\title{
Human/Computer interaction to learn scenarios from ICU multivariate time series
}

\author{
Thomas GUYET ${ }^{1}$, Catherine GARBAY ${ }^{1}$, and Michel DOJAT ${ }^{2}$ \\ 1 Laboratoire TIMC, 38706 La Tronche, France \\ thomas.guyet@imag.fr and http//www-timc.imag.fr/Thomas. Guyet/ \\ ${ }^{2}$ Mixt Unit INSERM/UJF U594
}

\begin{abstract}
In the context of high dependent environments such as intensive care or operating room we would like to predict, as soon as possible, from past and present states, the evolution of the patient's condition.

Based on the set of physiological dat, clinicians have difficulties to formalize typical high level scenes that are representative of specific patient's state sequences to recognize. On the other hand, signal processing algorithms are limited to low level pattern detection. We propose an interactive environment for an in-depth exploration by the clinician of data. We hypothesize that such human-computer collaboration could help with the definition of signatures representative of specific situations. To test our hypothesis, we have defined a multi-agent system $(M A S)$ with the capacities of 1) segmenting, 2) classifying and 3) learning. These three tasks are continuously adapted based on interactions with the clinician. Preliminary results are presented to support our assumption.
\end{abstract}

\section{Introduction}

Physiological monitors currently used in high dependency environments such as Intensive Care Units (ICUs) or anaesthesia wards generate a false alarm rate that approximately reaches $86 \%$ [1]. This bad score is essentially due to the fact that alarm detection is mono-parametric and based on predefined thresholds. To really assist clinicians in diagnosis and therapy tasks we should design intelligent monitoring systems with capacities of predicting the evolution of the patients state and eventually of triggering alarms in case of probable critical situations.

Several methods have been proposed for false alarm reduction based on data processing algorithms (using trend calculation) $[2,3]$ or data mining techniques [1]. In general, the methods proposed are based on two steps, first data processing algorithms detect relevant events in time series data and secondly information analysis techniques are used to identify well-known critical patterns. When applied to multivariate time series, these methods can be powerful if patterns to discover are well-known in advance.

In order to predict the evolution of the patient's state, typical high level scenes, combination of several patterns that are representative of specific situations, have to be formalized. Unfortunately, clinicians have some difficulties in defining such situations. We propose an interactive environment for an in-depth 
exploration by the clinician of the set of physiological data. In the interactive environnement TSW, used in [4], allows to collect overall statistics. Our approach is based on a collaborative work between a computer, which efficiently analyzes a large set of data and classifies information, and a clinician, who has skills and pragmatic knowledge for data interpretation and drives the computerized data exploration. We assume that such a collaboration could facilitate the discovery of signatures representative of specific situations. To test this hypothesis we have designed a collaborative multi-agent system $(M A S)$ with the capacities of segmenting, classifying and learning.

\section{Scenarios construction based on clinician/computer interaction}

The philosophy of the approach is the creation of a self-organized system guided by an clinician. The system dynamically builds its own data representation for scenario discovery. The clinician interacts with the system in introducing annotations, solving ambiguous cases, or introducing specific queries. Consequently, the system should be highly adaptive.

Data, information and knowledge are dynamically managed at several steps during clinician/computer interactions. Data, numeric or symbolic, are represented as multivariate time series. The whole system is an experimental workbench. The clinician can add or delete pieces of information, explore time series by varying the time granularity, using trends computed on various slidingwindows, and in selecting or combining appropriate levels of abstraction. Annotations can be introduced by the clinician generally as binary information (for instance, cough (0 or 1$)$, suction (0 or 1$)$ ).

The goal of our method is to learn from time series data the more likely scenario, i.e. a set of events linked by temporal constraints, that explains a specific event. In the following, we distinguish between events, that contribute to the description of a scenario, and specific events that are explained by scenarios. Specific events are characterized by a decision tree that is built from "instantaneous" patient states described by numeric and symbolic time series data and annotated as positive or negative examples. Then the decision tree is used to find new occurrences of the same type of specific events. To explain the so called specific events, scenarios are learned by the MAS (cf. section 3).

A clinician interacts with the $M A S$ to guide the learning and be informed of discoveries. A short example can briefly describe our method

1) Starting from time series data, the clinician annotates some $\mathrm{SpO}_{2}$ desaturation episodes in specific regions of interest. Annotations are encoded as binary time series data.

2) From data plus annotations, the system learns desaturation episodes, their characteristics (a tree) and possible scenario (learned by the $M A S$ ) that explains these episodes (specific events).

3) The system, in browsing a part of time series data, searches for similar characteristics and scenario. 
4) Based on similar characteristics, new desaturation episodes can be discovered by the system. They are used as new examples for the decision tree or if they present an atypical signature compared to others, they are shown to the clinician for examination.

5) When similar scenarios not followed by a desaturation episode are discovered, they are provided to the clinician who decides either to keep or reject them. They are then used respectively as positive or negative examples for the learning phase

6) The system loops to step 2 until no new scenarios or characteristics can be discovered.

Depending on its discoveries and information provided by the clinician, the system continuously adapts its behavior. Its complex self-organisation is masked to the clinician who interacts with the system only via time series data.

\section{A Multiagent System}

MASs present good properties to implement self-organization and reactivity to user interactions. Our general architecture was inspired by [5]. Three data abstraction levels, namely segments, classes of segments and scenarios, are constructed in parallel with mutual and dynamic adaptations to improve the final result. These levels are associated to a numerical, a symbolic and a semantic level respectively.

The MAS ensures an equivalence between what we call specific events and classes of segments. For symbolic time series, like annotations, symbols are associated to some classes of segments. For instance, the clinician can annotate each $\mathrm{SpO}_{2}$ desaturation episode. Learning a scenario that explains this specific event is equivalent to learning a scenario that explains occurrences of a class of segments (with the corresponding symbol) in the time series "SpO 2 annotations". Other classes of segments discovered by the system should be interpreted by the clinician.

Three types of agents exist in the MAS. At the numerical level, reactive agents segment time series. Each reactive agent represents a segment, i.e. a time series region bounded by two borders with neighboring agents. Frontiers move, disappear or are created dynamically by interactions between agents based on the approximation error of an $S V R^{3}$ model of segment. Then, at the symbolic level, classification agents build classes of segments. To compare segments, we have defined a distance between $S V R$ models that takes into account dilatation and scaling. A vocabulary is then introduced to translate times series into symbolic series.

At the semantic level, learning agents build scenarios that explain classes of segments (or specific events for clinicians). In fact, we consider a scenario that occurs before a specific event $e_{2}$ as a possible explanation for $e_{2}$. Consequently, learning a scenario that explains $e_{2}$ consists in finding common points (signature) in temporal windows preceding examples of the class of $e_{2}$. To find such

\footnotetext{
${ }^{3}$ Support Vector Regression [6]
} 
signatures, we have adapted the algorithm presented in [7] to multivariate series inputs. This algorithm takes into account temporal constraints to aggregate frequent relevant information and to progressively suppress noisy information. Then, the learning agent proposes modifications (via feedback) to segmentation and classification agents by focussing on discrepancies found between the learned scenario and the examples. In this way, it improves its global confidence.

Interactions are the key of the self-organization capability of the system and are used at three main levels

1) Feedback that dynamically modifies, adds or deletes segmentation agents, thus leading to segmentation modifications.

2) Feedback that adjusts classification.

3) Improvement of the overall learning accuracy (discrimination power) by pointing out, between learning agents, inconsistencies in the resulting associations of scenarios and specific events.

We highlight that at the two first levels, symbolization of all series are performed independently. However, learning agents use multivariate symbolic series to build scenarios and to deliver feedback to obtain a global coherence.

\section{Preliminary results}

We used data from ICU patients under weaning from mechanical ventilation. We selected four physiological signals $\left(\mathrm{SpO}_{2}\right.$, Respiratory rate, Total expired volume and heart rate) sampled at $1 \mathrm{~Hz}$ during at least 4 hours. Each signal was preprocessed by computing sliding-windows means (3 min width) and was abstracted by following a specific methodology [8] into symbolic values and trends. Moreover, we used $\mathrm{SpO}_{2}$ desaturation annotations inserted at the patient's bedside by a clinician. Consequently, each patient's recordings was a multivariate time series that contained $17(4 * 4+1)$ long times series. Presently, the implementation of our $M A S$ is partial, feedbacks are not fully implemented. Data acquisition is still undergoing. Thus, the quantity of data currently available is not sufficient to ensure a robust learning.

The figure 1 displays the experimental computer's interface to manage the $M A S$. The panel A) shows the way to access to patient's data and agents classes. The Panel $\mathrm{B}$ ) shows recordings for 2 patients $(P 1$ and $P 2)$. For each one, we display two time series "Heart Rate data" (numeric) and "SpO $\mathrm{O}_{2}$ Annotations" (symbolic). Vertical bars represent frontiers of segments obtained by segmentation agents. $\mathrm{SpO}_{2}$ annotations are segmented in 3 (for $\mathrm{P} 1$ ) and 5 (for P2) segments. Segmentation is not perfect especially for heart rate but will be refined by feedback. In panel $\mathrm{C}$ ), are displayed the 2 classes of segments that classify the 8 segments (only 7 are shown) of " $\mathrm{SpO}_{2}$ Annotations" time series. In panel D) the more probable scenario that explains class 2 is indicated as a set of events linked by temporal relations. A clinician interface should be developed to mask technical aspects of this interface and translate scenarios into comprehensible representations. 


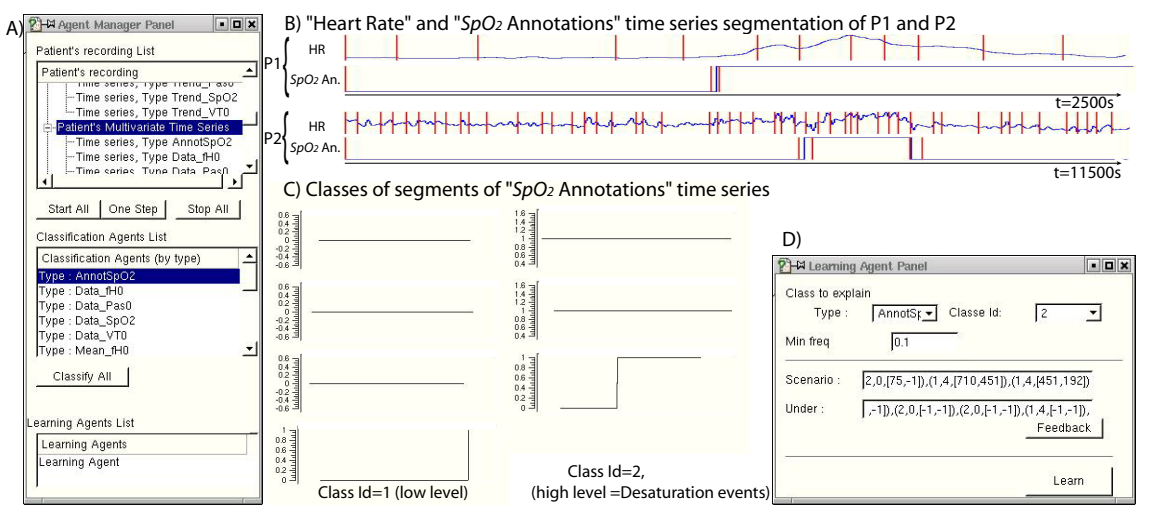

Fig. 1. $M A S$ interface. (see text for details)

\section{Conclusion and perspectives}

The system we present proposes an experimental workbench to assist clinicians in the exploration of physiological data in poorly formalized domains such as ICUs. The central concept of our system is to support interactions between a selforganized $M A S$, with data processing, data abstraction and learning capabilities and a clinician, who browses the provided mass of data to guide the learning of scenarios representative of the patient's state evolution. Preliminary results invite us to pursue the implementation and show the necessity to reinforce the $M A S$ capabilities to segment, classify and learn from time series data.

\section{References}

1. Tsien, C.L., Kohane, I.S., McIntosh, N.: Multiple signal integration by decision tree induction to detect artifacts in the neonatal intensive care unit. Art. Intel. in Med. 19 (2000) 189-202

2. Salatian, A., Hunter, J.: Deriving trends in historical and real-time continuously sampled medical data. J. Intel. Inf. Syst. 13 (1999) 47-71

3. Lowe, A., Harrison, M.J., Jones, R.W.: Diagnostic monitoring in anaesthesia using fuzzy trend templates for matching temporal patterns. Art. Intel. in Med. 16 (1999) 183-199

4. Hunter, J., Ewing, G., Freer, Y., Logie, F., McCue, P., McIntosh, N.: Neonate: Decision support in the neonatal intensive care unit - a preliminary report. In: AIME. (2003) 41-45

5. Heutte, L., Nosary, A., Paquet, T.: A multiple agent architecture for handwritten text recognition. Pattern Recognition 37 (2004) 665-674

6. Vapnik, V., Golowich, S., Smola, A.: Support vector method for function approximation, regression estimation, and signal processing. Ad. in Neural Inf. Proc. Sys. 9 (1997) 281-287

7. Dousson, C., Duong, T.V.: Discovering chronicles with numerical time constraints from alarm logs for monitoring dynamic systems. In: IJCAI. (1999) 620-626

8. Silvent, A.S., Dojat, M., Garbay, C.: Multi-level temporal abstraction for medical scenarios construction. Int. J. of Adaptive Control Signal Proc. (2005) Processing to appear. 